\title{
Dinâmica espacial da paisagem do Parque Estadual do Jalapão (TO) de 2000 a 2015
}

\author{
Space dynamics of the landscape of the State Park of Jalapão (TO) from 2000 to \\ 2015
}

\author{
Jader Nunes Cachoeira', Allan Deyvid Pereira da Silva"I, \\ Antonio Carlos Batista ${ }^{\mathrm{III}}$, Daniela Biondi ${ }^{\mathrm{IV}}$, Marcos Giongo ${ }^{\mathrm{V}}$, \\ Eduardo Ganassoli Neto ${ }^{\mathrm{VI}}$
}

\begin{abstract}
Resumo
A modificação da paisagem e a fragmentação do habitat tornaram-se grandes temas de pesquisa, uma vez que são consideradas ameaças graves para a biodiversidade. Considerando o histórico de perturbação da área do Parque Estadual do Jalapão (PEJ), dado sua característica de Unidade de Conservação (UC) de proteção integral inserida no bioma Cerrado, o objetivo desta pesquisa é atualizar o mapa de uso e cobertura do solo dessa UC e analisar as métricas que quantificam a conectividade do habitat e a fragmentação da ecologia da paisagem para o ano de 2015, tendo como base o mapa de uso e ocupação do solo do ano 2000. Para elaborar o mapa de uso do solo do PEJ de 2015, utilizou-se a cena 221/67 (órbita/ponto) de 8 de agosto de 2015 do satélite Landsat 8, sensor OLI, por abranger toda a área de estudo e por não ter cobertura de nuvens. No processamento digital das imagens foram realizadas a correção radiométrica, a pré-classificação e a associação das classes de uso do solo, e, posteriormente, classificação supervisionada por meio de interpretação visual. Para verificar a precisão do mapa foi calculado o coeficiente de Kappa, cujo valor foi de 0,92 . A partir do mapa atualizado foram realizados os cálculos de índices de ecologia da paisagem referentes à área, tamanho, forma, borda e proximidade dos fragmentos. Os procedimentos foram realizados no software SIG ArcGIS 10.4 por meio da extensão gratuita Patch Analyst 5.2. Com base nos mapas de uso e cobertura do solo e nos índices de ecologia da paisagem, pode-se constatar que houve mudanças na paisagem. Quanto às métricas, conclui-se que a UC se encontra fragmentada, apresenta 564 fragmentos e possui alta heterogeneidade espacial e forma complexa e irregular em razão dos valores de MPS, PSCoV e MSI.
\end{abstract}

Palavras-chave: Uso do solo; Métricas; Conservação; Ecologia da Paisagem

Advogado, Me., Doutorando do Programa de Pós-graduação em Engenharia Florestal, Universidade Federal do Paraná, Avenida Lothário Meissner, 632, CEP 80210-170, Curitiba (PR), Brasil. jadernunes@uft.edu.br (ORCID: 0000-0001-9150-5431)

II Geógrafo, Me., Doutorando do Programa de Pós-graduação em Engenharia Florestal, Universidade Federal do Paraná, Avenida Lothário Meissner, 632, CEP 80210-170, Curitiba (PR), Brasil. allanuft@gmail.com (ORCID: 0000-0003-4424-0463)

III Engenheiro Florestal, Dr., Professor do Departamento de Ciências Florestais, Universidade Federal do Paraná, Avenida Lothário Meissner, 632, CEP 80210-170, Curitiba (PR), Brasil. batistaufpr@gmail.com (ORCID: 0000-0001-5929-3838)

IV Engenheira Florestal, Dra., Professora do Departamento de Ciências Florestais, Universidade Federal do Paraná, Avenida Lothário Meissner, 632, CEP 80210-170, Curitiba (PR), Brasil. dbiondi@ufpr.br (ORCID: 0000-0003-0532-7363)

Engenheiro Florestal, Dr., Professor do Curso de Engenharia Florestal, Universidade Federal do Tocantins, Rua Badejós, chácaras 69 e 72 , Zona Rural, CEP 77404-970, Gurupi (TO), Brasil. giongo@uft.edu.br (ORCID: 0000-0003-1613-6167)

vi Engenheiro Florestal, Me., Pesquisador Autônomo, Av. França, 218, CEP 77440-640, Gurupi (TO), Brasil. engf.edu@gmail.com (ORCID: 0000-00029282-2698) 


\begin{abstract}
Landscape modification and habitat fragmentation have become major research topics. They are considered serious threats to biodiversity. The disturbance history of the area of Jalapão State Park (Parque Estadual do Jalapão - PEJ) was analysed given its characteristic as a Conservation Unit (CU) of integral protection inserted into the Cerrado biome. Thus, the objective of this research is to update the map of land use and cover of this CU and analyse the metrics that quantify habitat connectivity and fragmentation in the landscape ecologyfor 2015 based on the land use map for 2000. In order to plot the PEJ's 2015 map of land use, we used the scene 221/67 (orbit/point) of August 8, 2015, from the Landsat 8 satellite, OLI sensor, since it covered the entire study area and did not present cloud cover. In the digital image processing, radiometric correction, pre-classification, and association of classes of soil use were performed. Subsequently, a supervised classification was performed through visual interpretation. To verify map accuracy, the Kappa coefficient was calculated. Its value was 0.92 . From the updated map, calculations of landscape ecology indexes were performed considering area, size, shape, border, and proximity of fragments. The procedures were performed using the software GIS ArcGIS 10.4 and the free extension Patch Analyst 5.2. Based on the maps of land use and cover, and indexes of landscape ecology, it is possible to verify that there were changes in the landscape. As for metrics, it can be concluded that the CU is fragmented (564 fragments) and has a high spatial heterogeneity, a complex form, and an irregular shape due to values of MPS, PSCoV and MSI.
\end{abstract}

Keywords: Land use; Metrics; Conservation; Landscape Ecology

\title{
Introdução
}

O estudo da paisagem deve considerar três características principais: sua estrutura, formada por uma matriz (elemento predominante), fragmentos e corredores; sua função, relacionada à interação entre os elementos que a compõem e sua dinâmica, as alterações na estrutura e função ao longo do tempo (FORMAN; GODRON, 1986).

A fragmentação de habitats acarreta isolamento das formações e populações remanescentes, alterações nos fluxos gênicos, intensificação das competições, alterações da estrutura e qualidade de habitats e extinções de espécies (MUCHAILH et al., 2010). Jesus et al. (2015) afirmaram que a aceleração do processo de fragmentação causa a sua diminuição, tornando-as suscetíveis ao efeito de borda. Assim, é importante avaliar aspectos tais como estrutura, diversidade e composição dos ambientes para obter informações com a finalidade de escolher a melhor opção nas medidas de restauração ou conservação de áreas degradadas ou alteradas que são produtos da fragmentação (MUCHAILH et al., 2010).

Uma das formas mais utilizadas para se analisar a fragmentação de paisagens é através da aplicação de métricas da paisagem por possuírem aplicações importantes na quantificação do crescimento ou da expansão da fragmentação da paisagem. Para Frohn e Hao (2006), tais métricas do padrão de paisagem são usadas para quantificar a composição e a configuração da paisagem em um mapa ou imagem de sensoriamento remoto. As técnicas de geoprocessamento e de processamento digital de imagens permitem a avaliação das alterações do ambiente, viabilizando a compreensão dos padrões de organização espacial, com os quais é possível diagnosticar e até mesmo prognosticar as mudanças da paisagem e mostrar a distribuição espacial real das diferentes formas de uso dos recursos naturais cada vez mais alterados pelas ações do homem (RUDOLPHO et al., 2013).

Para as Unidades de Conservação (UCs) abertas ao público e com presença de moradores e extrativistas (comunidades tradicionais), somadas às pressões da expansão da fronteira agrícola no entorno, como é o caso do Parque Estadual do Jalapão (PEREIRA JÚNIOR et al., 2014), justifica-se o presente estudo, cujo objetivo é atualizar o mapa de uso e cobertura da terra e analisar o processo de fragmentação da paisagem por meio de métricas da paisagem. 


\section{Material e métodos}

\section{Local de estudo}

O Parque Estadual do Jalapão (PEJ) está na porção nuclear da região do Jalapão, inteiramente dentro no município de Mateiros, no leste do Tocantins, com área de 159.539,7 ha, no retângulo formado pelos paralelos $10^{\circ} 08^{\prime} 53^{\prime \prime}$ e $10^{\circ} 36^{\prime} 32^{\prime \prime}$ S e entre os meridianos $46^{\circ} 24^{\prime} 24^{\prime \prime}$ e $46^{\circ} 56^{\prime} 06^{\prime \prime} \mathrm{W}$.

O Parque possui formações campestres, savânicas e florestais. As formações campestres são campo limpo, campo limpo úmido e campo sujo; as savânicas são as diferentes formações do cerrado em sentido restrito (típico, ralo, denso, rupestre) e a vereda; e as florestais são o cerradão, as matas de galeria não inundável e inundável, a mata ciliar e a mata seca semidecídua. A geomorfologia é caracterizada por planaltos e chapadões aplainados, sendo observados serras do tipo "mesa" e morros "testemunhos" com bordas ruiniformes. Predominam os solos neossolos quartzarênicos e litólicos e os latossolos vermelhos (TOCANTINS, 2003).

A maior parte da economia da região está centrada em agricultura de subsistência, pecuária extensiva, artesanato de capim-dourado e turismo. Essas atividades econômicas principais dependem diretamente do cerrado e, com exceção do turismo, todas envolvem o uso de fogo (SCHMIDT et al., 2011).

\section{Mapa de uso do solo}

Para a elaboração do presente estudo foram utilizados arquivos vetoriais do uso e cobertura da terra do ano 2000, disponibilizados pela Secretaria de Planejamento do Estado do Tocantins (TOCANTINS, 2012). A partir desses dados, o mapa de uso e ocupação do solo do PEJ foi atualizado para 2015. Para tanto, foram adquiridas imagens multiespectrais do sensor OLI (Operacional Land Imagem) a bordo do satélite Landsat-8 (Land Remote Sensing Satellite) constante do acervo de cenas do United States Geological Survey (USGS), disponível de modo ortorretificado na plataforma GLOVIS (www.glovis.usgs.gov).

Foi utilizada a cena 221/67 (órbita/ponto), de 8 de agosto de 2015, para obter o mosaico. A data de passagem do satélite foi escolhida de acordo com o período de estiagem na região e com menor quantidade de nuvens ( $5 \%$ de presença de nuvens). As duas primeiras fases do processamento digital da imagem, pré-processamento e processamento, foram executadas seguindo a metodologia de Xaud e Epiphanio (2014). Na fase de classificação, adotou-se o método da classificação supervisionada por interpretação visual, que consiste na identificação das classes de informação (tipos de cobertura da terra) presentes na imagem e posterior caracterização das refletâncias para cada classe de informação.

A variação da área ocupada por cada classe entre os anos 2000 e 2015 foi obtida por meio da seguinte relação: [(A2015 - A2000) / A2000] * 100, em que A = área ocupada (ha) por determinada classe no ano indicado.

Para a classificação da imagem referente a 2015, foram coletados 133 pontos de controle em toda a área de estudo para inferir a exatidão das classes. Cada ponto foi associado à classe presente no local e, posteriormente, essas informações foram cruzadas com mapeamento realizado para 2015, sendo gerada a matriz de confusão entre os pontos controle e a classificação realizada a fim de obter o índice de Kappa.

O desempenho do índice de Kappa foi avaliado de acordo com a tabela de desempenho proposta por Fonseca (2000). Índices entre 0,21 e 0,4 são considerados razoáveis, entre 0,41 e 0,6 bons, entre 0,61 e 0,8 muito bons, e entre 0,81 e 1 excelentes. 


\section{Métricas da paisagem}

Este estudo integrou geotecnologias (Sensoriamento Remoto e SIG) e métricas espaciais para analisar a dinâmica espaço-temporal e a evolução da mudança de uso da terra e padrão de paisagem. Indicadores geoespaciais eficientes para uma melhor compreensão do processo de fragmentação da paisagem do PEJ foram obtidos.

A partir do mapa de uso do solo foi realizado o cálculo de índices de ecologia da paisagem referentes à área, tamanho, forma, borda e proximidade conforme as descrições constantes na Tabela 1. Os procedimentos foram realizados no software SIG ArcGIS 10.4 por meio da extensão gratuita Patch Analyst 5.2, desenvolvida por Rempel, Kaukinen e Carr (2012).

\section{Tabela 1 - Métricas da paisagem obtidas ao nível de classes}

Table 1 - Landscape metrics obtained at class level

\begin{tabular}{|c|c|c|c|c|}
\hline Grupo & Sigla & Métrica & Unidade & Observação \\
\hline Área & CA & Área da Classe & Hectare (ha) & $\begin{array}{l}\text { Somatório das áreas de todos os } \\
\text { fragmentos presentes na área. }\end{array}$ \\
\hline \multirow{3}{*}{$\begin{array}{l}\text { Densidade e } \\
\text { tamanho }\end{array}$} & MPS & $\begin{array}{l}\text { Tamanho médio do } \\
\text { fragmento }\end{array}$ & Hectare (ha) & $\begin{array}{l}\text { Soma do tamanho dos fragmentos } \\
\text { dividido pelo número. }\end{array}$ \\
\hline & NUMP & Número de fragmentos & Adimensional & $\begin{array}{c}\text { Número total de fragmentos na } \\
\text { paisagem/classe. }\end{array}$ \\
\hline & PSCoV & $\begin{array}{c}\text { Coeficiente de variação } \\
\text { do tamanho do } \\
\text { fragmento }\end{array}$ & Porcentagem (\%) & $\begin{array}{l}\text { Desvio padrão do tamanho do } \\
\text { fragmento dividido por seu tamanho } \\
\text { médio, multiplicado por } 100 \text {. }\end{array}$ \\
\hline \multirow{2}{*}{ Borda } & $\mathrm{TE}$ & Total de bordas & Metro (m) & $\begin{array}{l}\text { Soma do perímetro de todos os } \\
\text { fragmentos. }\end{array}$ \\
\hline & ED & Densidade de borda & $\mathrm{m} / \mathrm{ha}$ & $\begin{array}{l}\text { Quantidade de extremidades relativa } \\
\text { à área da paisagem. }\end{array}$ \\
\hline \multirow[t]{2}{*}{ Forma } & MSI & Índice de forma médio & Adimensional & $\begin{array}{c}\text { É igual a um quando os fragmentos } \\
\text { forem circulares e aumenta o } \\
\text { valor do índice com a crescente } \\
\text { irregularidade da forma do } \\
\text { fragmento. }\end{array}$ \\
\hline & AWMSI & $\begin{array}{l}\text { Índice de forma de } \\
\text { área média ponderada }\end{array}$ & Adimensional & $\begin{array}{c}\text { Difere do MSI, porque fragmentos } \\
\text { maiores terão mais peso que as } \\
\text { menores. }\end{array}$ \\
\hline Proximidade & MNN & $\begin{array}{l}\text { Distância média do } \\
\text { vizinho mais próximo }\end{array}$ & $\operatorname{Metros}(\mathrm{m})$ & $\begin{array}{l}\text { A distância média do vizinho mais } \\
\text { próximo é a média dessas distâncias } \\
\text { para classes individuais no nível de } \\
\text { classe e a distância média da classe } \\
\text { vizinha mais próxima no nível de } \\
\text { paisagem. }\end{array}$ \\
\hline
\end{tabular}

Fonte: Adaptado de MacGarigal e Marks (1995) 


\section{Resultados e discussão}

\section{Uso do Solo}

A partir da matriz de confusão foi obtido o índice de Kappa $(\mathrm{k})$ que, para o uso do solo do PEJ de 2015, foi de 0,92, confirmando a eficiência da metodologia aplicada no presente trabalho (FONSECA, 2000).

$\mathrm{Na}$ análise dos mapas de uso do solo (Figura 1), notam-se algumas alterações na paisagem do PEJ. Os valores de área em hectares (ha) ocupados por cada classe nos anos 2000 e 2015 e os valores das variações (\%), também de cada classe, encontram-se na Tabela 2.

Figura 1 - Mapa temático da cobertura do solo do PEJ em 2000 (A) e 2015 (B)

Figure 1 - Thematic map of PEJ soil cover in 2000 (A) and 2015 (B)

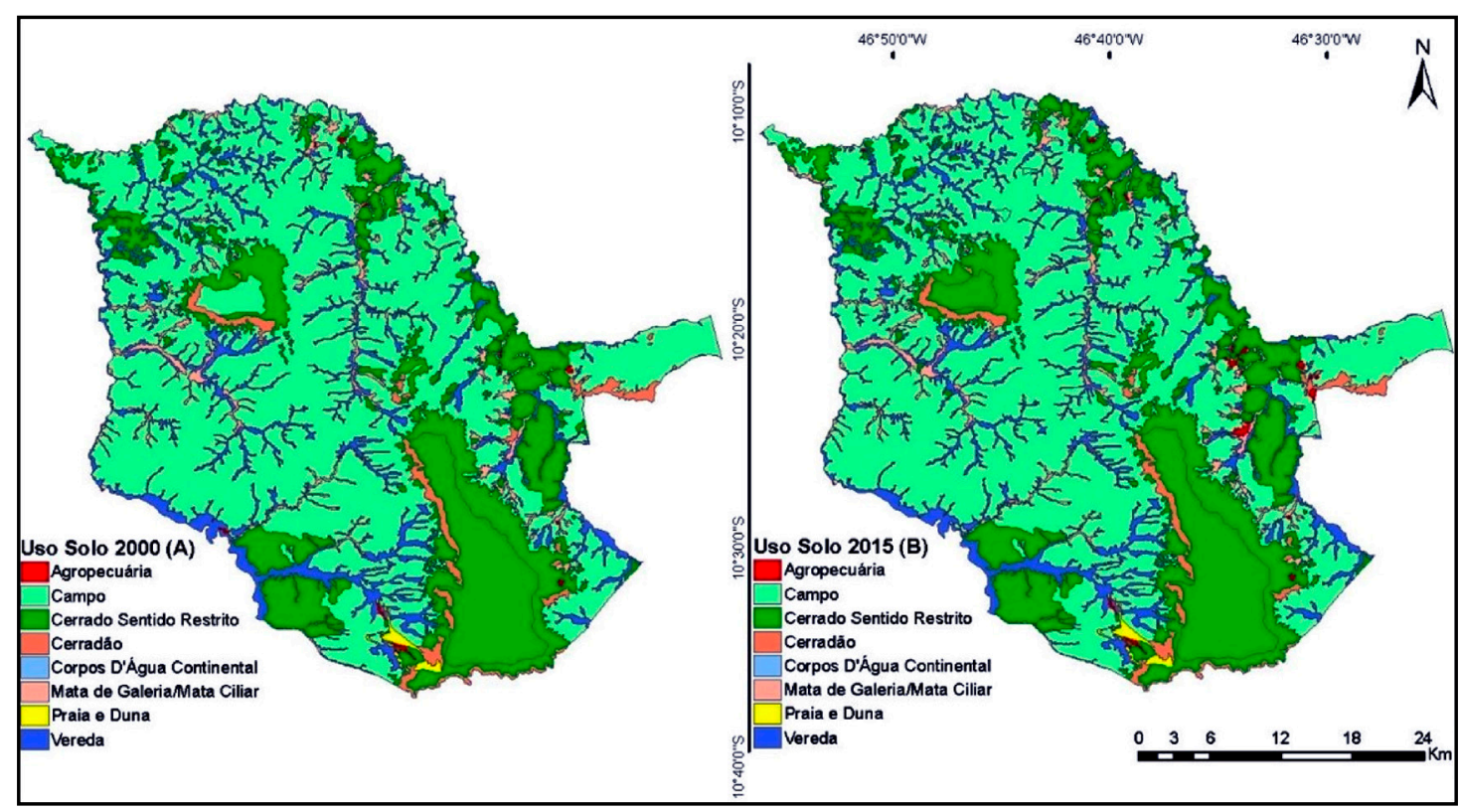

Fonte: Autores (2018)

Depreende-se da Tabela 2 que o PEJ possuía, tanto em 2000 quanto em 2015, a maior parte de sua área coberta por Campo, 60,31\% (2000) e 58,38\% (2015), que compreende as fitofisionomias Campo Limpo e Campo Sujo, seguido pela classe Cerrado Sentido Restrito, que cobria 23,59\% da área do PEJ no ano 2000 e 25,17\% em 2015.

As áreas de Agropecuária foram as que mais apresentaram variação no período de 2000 a 2015, isto é, $114,89 \%$ de acréscimo. A Figura 1 demonstra onde essas áreas estão localizadas, notadamente na região noroeste, mais próximas ao município de São Félix (TO), e a maior concentração na região leste, onde se localiza a sede do município de Mateiros (TO). O aumento das áreas de Agropecuária e, consequentemente, a diminuição das áreas de Campo e Cerradão (Tabela 2) pode estar relacionada à intensificação do uso do solo pelos moradores e agricultores familiares residentes no PEJ como consequência a problemas quanto às desapropriações na época da criação do Parque (TOCANTINS, 2003; PEREIRA JÚNIOR et al., 2014). 


\section{Tabela 2 - Área ocupada por cada classe de uso do solo no Parque Estadual do Jalapão nos anos de 2000 e 2015}

Table 2 - Area occupied by each class of land use in Jalapão State Park in 2000 and 2015

\begin{tabular}{|c|c|c|c|c|c|}
\hline \multirow{2}{*}{ Classes de uso } & \multicolumn{2}{|c|}{2000} & \multicolumn{2}{|c|}{2015} & \multirow{2}{*}{$\begin{array}{c}\text { Variação } \\
(2000-2015)\end{array}$} \\
\hline & Área (ha) & Área (\%) & Área (ha) & Área (\%) & \\
\hline Agropecuária & 276,23 & $0,17 \%$ & 593,59 & $0,37 \%$ & $114,89 \%$ \\
\hline Campo & $96.218,36$ & $60,31 \%$ & $93.139,36$ & $58,38 \%$ & $-3,20 \%$ \\
\hline Cerradão & $3.674,03$ & $2,30 \%$ & $3.532,03$ & $2,21 \%$ & $-3,86 \%$ \\
\hline Cerrado Sentido Restrito & $37.634,55$ & $23,59 \%$ & $40.159,97$ & $25,17 \%$ & $6,71 \%$ \\
\hline Corpos d'água & 261,83 & $0,16 \%$ & 184,97 & $0,12 \%$ & $-29,35 \%$ \\
\hline Mata de Galeria/Mata Ciliar & $5.482,06$ & $3,44 \%$ & $5.668,78$ & $3,55 \%$ & $3,41 \%$ \\
\hline Praia e Duna & 504,81 & $0,32 \%$ & 504,81 & $0,32 \%$ & $0,00 \%$ \\
\hline Vereda & $15.491,84$ & $9,71 \%$ & $15.756,00$ & $9,88 \%$ & $1,71 \%$ \\
\hline
\end{tabular}

Fonte: Autores (2018)

Dentre as classes que mais variaram quanto à redução de áreas, os Corpos d'água apresentaram perda de $29,35 \%$. Essa variação pode estar relacionada com a quantidade de precipitação (chuvas) nos períodos de estudo. Dados da estação meteorológica de Mateiros (TO), disponibilizados pela Agência Nacional de Águas (AGÊNCIA NACIONAL DE ÁGUAS, 2017), informam que nos anos de 1999 e 2000 o acumulado de precipitação $(\mathrm{mm})$ foi de $2.824,2$ e $3.487,2$, respectivamente, ao passo que nos anos de 2014 e 2015 a quantidade de chuvas foi bem menor: 1.035,7 mm em 2014 e 1.075,4 mm em 2015. Ou seja, no mapeamento em 2000 houve um acúmulo maior de água, que culminou em uma maior delimitação de áreas classificadas como Corpos d'água, ao passo que em 2015 ocorreu uma diminuição da quantidade ou duração do período de chuvas, resultando em uma menor identificação e delimitação dessa classe.

$\mathrm{O}$ aumento das áreas de Mata de Galeria e Veredas está provavelmente relacionado com a diminuição das áreas de Corpos d'água, que tiveram um comportamento inverso. Essas variações podem estar associadas ao fato de que essas duas classes se encontram em áreas úmidas (às margens de cursos d'água).

\section{Métricas da paisagem}

Forman e Godron (1986) e Pirovani et al.(2014) afirmam que a área é uma das informações mais importantes da paisagem, pois é a base para o cálculo de outras métricas, explicando as variações de riqueza de espécies. A Tabela 3 demonstra a variabilidade dos fragmentos florestais do PEJ, evidenciando que sua área de $159.539,7$ ha é formada por 524 fragmentos. Há desde fragmentos muito pequenos (menores que $1 \mathrm{ha}$ ) até fragmentos muito grandes (>15.000 ha).

A classificação dos fragmentos por tamanho de área, como se verifica na Tabela 3 , mostra como a paisagem do PEJ apresenta algum grau de fragmentação. Observa-se que na área de estudo são encontradas classes de fragmentos muito pequenos, sendo que 83 fragmentos representam $15,8 \%$ do total, perfazendo tão somente $0,1 \%$ da área da paisagem. Destes, 21 fragmentos possuem área menor do que 1 ha e $10,9 \%$ (57) do total de fragmentos foram classificados como pequenos $(5,01-10$ ha). Somadas as áreas, representam $0,3 \%$ ( 411,35 ha) do total da área. Na classe de médios fragmentos (10,01-100 ha) há 269 fragmentos (51,34\%), com área de 10.483,25 ha, representando apenas 6,6\% da área total da paisagem do PEJ. 


\section{Tabela 3 - Número de fragmentos por classe de área do Parque Estadual do Jalapão}

Table 3 - Number of fragments per area class of the Jalapão State Park

\begin{tabular}{|c|c|c|c|c|}
\hline \multirow{2}{*}{ Classes de área (ha) } & \multicolumn{2}{|c|}{ Quantidade de fragmentos } & \multicolumn{2}{|c|}{ Área das classes } \\
\hline & Und & $\%$ & Ha & $\%$ \\
\hline$<5$ & 83 & $15,80 \%$ & 176,53 & $0,10 \%$ \\
\hline $5,01-10$ & 57 & $10,90 \%$ & 411,36 & $0,30 \%$ \\
\hline $10,01-20$ & 70 & $13,40 \%$ & 998,02 & $0,60 \%$ \\
\hline $20,01-40$ & 94 & $17,90 \%$ & $2.736,70$ & $1,70 \%$ \\
\hline $40,01-60$ & 56 & $10,70 \%$ & $2.760,40$ & $1,70 \%$ \\
\hline $60,01-100$ & 49 & $9,40 \%$ & $3.988,13$ & $2,50 \%$ \\
\hline $100,01-200$ & 45 & $8,60 \%$ & $6.107,87$ & $3,80 \%$ \\
\hline $200,01-600$ & 42 & $8,00 \%$ & $12.752,65$ & $8,00 \%$ \\
\hline $600,01-1000$ & 10 & $1,90 \%$ & $7.093,21$ & $4,40 \%$ \\
\hline $1.000,01-15.000$ & 17 & $3,20 \%$ & $66.059,00$ & $41,40 \%$ \\
\hline$>15.000$ & 1 & $0,20 \%$ & $56.455,65$ & $35,40 \%$ \\
\hline
\end{tabular}

Fonte: Autores (2018)

Em que: Quantidade de fragmentos: Und, os valores 70, 94, 56 e 49 somam 269; \%, os valores $13,40 \%, 17,90 \%, 10,70 \%$ e 9,40\% somam 51,34\%; Área de classes: $\%$, os valores $0,60 \%, 1,70 \%, 1,70 \%$ e $2,50 \%$ somam $6,50 \%$.

Quanto à classe dos fragmentos com área acima de 100 ha, optou-se por categorizá-los nas classes grande, muito grande e enorme. Assim, referindo-se a estas três últimas categorias, a paisagem do PEJ também é formada por 97 (18,51\%) fragmentos com área entre 100,01 até 1.000 ha, com somatório das áreas de 25.953,73 há, ou 16,3\% do total de área, bem como por 17 (3,2\%) fragmentos muito grandes (1.000,01-15.000 ha) que somam $41,4 \%$ do total da área (66.059 ha); por último, o único fragmento categorizado como enorme, tendo em vista que sua área é de $56.455,65$ ha, ocupa $35,4 \%$ da área total (Tabela 3).

A categorização das classes de fragmentos seguiu, com adaptações em detrimento do tamanho da área do PEJ, os parâmetros dos trabalhos de Juvanhol et al. (2011) e Esteves Peluzio, Fiedler e Zanetti (2015), os quais, ao estudarem remanescentes da Mata Atlântica, atribuíram as classes de tamanho como muito pequeno ( $<5 \mathrm{ha})$, pequeno (5-10 ha), médio (10-100 ha) e grande ( $>100 \mathrm{ha})$. No entanto, essa classificação não é padrão. Pirovani et al. (2014), estudando a fragmentação na bacia do rio Itapemirim, atribuíram como pequenos a classe $<5$ ha, como médios de 5 a 50 ha e como grandes a classe de fragmentos acima de 50 ha (> 50 ha).

Uma característica comum encontrada nesses trabalhos com o PEJ é a relação inversa entre o número de fragmentos e a área que eles ocupam. Ou seja, as classes de fragmentos pequenos, muito pequenos e médios apresentaram maior número de fragmentos, porém a soma de suas áreas representou o menor percentual da área total, enquanto que as classes dos fragmentos grandes, muito grande e enorme, em menor número, apresentaram, significativamente, as maiores áreas, responsáveis pela maior cobertura florestal em área.

Ao contrário das constatações de Pirovani et al. (2014), os resultados das quantidades de fragmentos por classes de tamanho apresentados para o PEJ indicam que a área está conservada, já que 51,34\% dos fragmentos são de tamanhos médios. No entanto, para algumas classes, a grande quantidade de pequenos fragmentos florestais pode estar associada ao problema de susceptibilidade da 
área a alterações na dinâmica da paisagem, sendo que a riqueza diminui quando a área do fragmento fica menor do que as áreas mínimas necessárias para a sobrevivência das populações (SANTOS et al., 2007).

Outras observações, no que tange às dimensões dos fragmentos, demonstram que, isoladamente, tamanhos das classes de fragmentos abaixo de 100 ha encontradas para o PEJ são inviáveis para a conservação de algumas espécies do cerrado das quais se conhece a área de vida, por exemplo, 1.096 ha a 2.180 ha (53 animais) para Tayassu pecari (FRAGOSO, 1998) e 18,5 ha para Callithrix penicillata (MIRANDA; FARIA, 2001).

Deve-se considerar, no entanto, que o conhecimento da capacidade dos mamíferos florestais neotropicais de se moverem entre os fragmentos é praticamente inexistente. Portanto, a discussão do tamanho mínimo da área florestal deve ser pautada com a limitação do conhecimento do potencial de circulação de espécies em florestas tropicais (ANDREAZZI; PIRES; FERNANDEZ, 2009). Esse aspecto se correlaciona com o que Vieira et al. (2003) afirmam no sentido de que fragmentos menores que 100 ha devem ser preservados, pois mantêm uma parcela significativa da biodiversidade de mamíferos e, principalmente, por possuírem importante função atuando como stepping stones, o que pode, para algumas espécies, facilitar os fluxos entre fragmentos, promovendo aumento no nível de heterogeneidade da matriz (ALMEIDA, 2008).

Quanto à paisagem, no $\mathrm{PEJ}$ o tamanho médio das manhas (MPS) foi de 304,47 ha, com coeficiente de variação do tamanho das manchas (PSCoV) de 863,64\% (Tabela 4). Essa alta variação está relacionada ao fato de que no PEJ há fragmentos menores do que 1 ha $(21$ fragmentos de áreas de Cerrado Sentido Restrito e Matas de Galeria/Cililar) até fragmentos com área superior a mil hectares (18 fragmentos, sendo dois fragmentos de Campos com área superior a dez mil ha) (Figura 1 e Tabela 3).

\section{Tabela 4 - Métricas da paisagem do Parque Estadual do Jalapão}

Table 4 - Landscape metrics of the Jalapão State Park

\begin{tabular}{lccccccccc}
\hline \multirow{2}{*}{ Índices $^{*}$} & \multicolumn{7}{c}{ Classes } \\
\cline { 2 - 10 } & $\mathbf{A g}$ & Cag & Cam & Ce & Csr & Mg & Pr & Ve & Todas \\
\hline CA (ha) & 593,95 & 185,13 & $93.207,17$ & $3.534,19$ & $40.185,92$ & $5.672,92$ & 505,11 & $15.767,33$ & $159.539,70$ \\
ZLAND & 6 & 1 & 10 & 5 & 25 & 19 & 1 & 33 & 100 \\
MPS (ha) & 19,16 & 61,71 & $1.726,06$ & 130,9 & 304,44 & 58,48 & 101,02 & 90,1 & 304,47 \\
NumP & 31 & 3 & 54 & 27 & 132 & 97 & 5 & 175 & 524 \\
PSCoV (\%) & 93,02 & 53,19 & 454,34 & 157,49 & 362,94 & 181,58 & 97,24 & 346,73 & 863,64 \\
TE (m) & 69.957 & 56.153 & 2.312 .675 & 216.062 & 1.249 .083 & 792.694 & 35.012 & 1.877 .876 & 6.607 .150 \\
ED (m/ha) & 0,44 & 0,35 & 14,49 & 1,35 & 7,83 & 4,97 & 0,22 & 11,77 & 41,41 \\
MSI & 1,532 & 6,256 & 2,405 & 1,824 & 1,937 & 2,767 & 2,108 & 2,97 & 2,48 \\
MPAR & 0,017 & 0,028 & 0,011 & 0,011 & 0,059 & 0,034 & 0,011 & 0,021 & 0,031 \\
AWMSI & 1,7 & 7,71 & 10,97 & 3,25 & 3,43 & 5,19 & 2,38 & 7,11 & 8,25 \\
MPFD & 1,29 & 1,43 & 1,29 & 1,27 & 1,32 & 1,36 & 1,3 & 1,36 & 1,33 \\
MNN & 9,9 & 0,03 & 28169,49 & 146,96 & 1328,24 & 18,98 & 67,95 & 202,58 & 3317,53 \\
\hline
\end{tabular}

Fonte: Autores (2018)

Em que: Ag = Agropecuária; Cag = Corpos d'água; Cam = Campo; Ce = Cerradão; Csr = Cerrado sentido restrito; $\mathrm{Mg}=$ Mata de galeria/ciliar; $\mathrm{Pr}=$ Praia/dunas; Ve = Veredas; CA = Área total da Classe; MPS = Tamanho médio do fragmento; NumP = Número de manchas; PSCoV = Coeficiente de variação do tamanho da mancha; TE = Total de bordas; $\mathrm{ED}$ = Densidade de borda; $\mathrm{MSI}$ = Índice de forma médio; AWMSI = Índice de forma de área média ponderada; $\mathrm{MNN}=$ Índice de proximidade. 
Os valores do MPS e do PSCoV obtidos para a paisagem no PEJ evidenciam, de acordo com Calegari et al. (2010) e Pirovani et al. (2014), a alta variabilidade, o que é comum em estudos de ecologia da paisagem. Os autores também observaram elevados coeficientes de variação em relação à área média dos fragmentos, indicando alta heterogeneidade espacial da paisagem, que no PEJ está associada principalmente aos valores do tamanho médio dos fragmentos (MPS) e aos coeficientes de variação (PSCoV) da Classe Campo (Cam), com MPS de 1.726,06 ha e PSCoV 454,34\%, em detrimento dos valores encontrados para MPS e PSCoV para as Classes de Agropecuária (Ag) e Mata de Galeria/Ciliar (Mg) (Tabela 4).

No entanto, apesar de haver uma alta heterogeneidade, deve-se considerar que as paisagens que apresentam menores valores para tamanho médio de fragmento (MPS) são as mais fragmentadas em função do número de fragmentos e da área ocupada pela cobertura vegetal. Em situações de fragmentação em uma unidade de área contínua, espécies que ocupam o interior do fragmento podem sofrer redução devido à perda de habitats (ESTEVES PELUZIO; FIEDLER; ZANETTI, 2015). Nesse sentido, pode-se inferir que as Classes Mg e Ve apresentam condição de fragmentação, ensejando maiores atenções quanto à conservação.

Nas lições de Frohn e Hao (2006), uma das métricas mais eficazes de medição da fragmentação da paisagem é o Índice de Maior Fragmento (LPI), que tem sido amplamente utilizado como um indicador de fragmentação da paisagem. Para o PEJ, o LPI foi de 35\%, correspondendo a um único fragmento da Classe Cam, o que por sua vez significa dizer que essa área representa a matriz da paisagem do $\mathrm{PEJ}$ e que mais de um terço da área não se encontra fragmentada. O valor encontrado do LPI para o PEJ, matriz da paisagem, possui relevante valor. Um maior valor favorece a presença de dispersores, polinizadores e propágulos, abastecendo fragmentos menores (MCGARIGA; MARKS, 1995).

Quanto às métricas de borda, o total de borda (TE) representa a soma do perímetro de todos os fragmentos. Em termos de cobertura vegetal, em que se excluem as classes Pr e Cag, o menor valor de TE foi obtido para a classe Ag, com 69.957 m, seguida da classe Ce, com 216.062 $\mathrm{m}$, e Mg, que obteve $792.694 \mathrm{~m}$ de bordas. De forma inversa, as classes Cam, Ve e Csr tiveram os maiores valores de TE (Tabela 4).

De acordo com a Tabela 4, os resultados de ED também demonstraram a mesma ordem de valores das classes apresentada para TE. Ag $(0,44 \mathrm{~m} / \mathrm{ha})$, Ce $(1,35 \mathrm{~m} / \mathrm{ha})$ e $\mathrm{Mg}(4,97 \mathrm{~m} / \mathrm{ha})$ obtiveram os menores valores de $\mathrm{ED}$, ao passo que maiores valores foram obtidos para as Classes Cam (14,49 m/ha), Ve (11,77 m/ha) e Csr (7,83 m/ha). Pirovani et al. (2014) e Juvanhol et al. (2011) nos informam que a diferença na quantidade de bordas, quando se considera a densidade, é devida aos valores de área ocupados por cada classe de tamanho dos fragmentos, mas que pode sofrer alterações devido à quantidade de fragmentos, visto que quanto maior o número de fragmentos de determinada classe, maior a densidade de borda (CALEGARI et al., 2010). Todavia, no presente estudo, essa tendência não ocorreu de maneira direta.

As classes com menores áreas (Ag, Ce e Mg) também apresentaram, proporcionalmente, os menores valores de TE e ED. Porém, quanto às classes com maiores áreas, somente a Classe Cam apresentou quantidades proporcionais de TE e ED. O predomínio dos valores de TE e ED da Classe Cam sobre as demais Classes se deve à maior área associada ao número de fragmentos desta Classe, comprovando que ela é a matriz da paisagem do PEJ. Entretanto, apesar de possuir área maior do que a da Classe Ve, a Classe Csr possui menos quantidade de TE e ED. Isso pode ter ocorrido devido a seu maior valor de área e menor quantidade de fragmentos e à compactação da Classe Ve, com elevado número de fragmentos e área menor, apontando para um menor efeito de borda e indicando um maior grau de conservação da Classe Csr (JUVANHOL et al., 2011; PIROVANI et al., 2014).

A forma dos fragmentos também influencia o grau do impacto do efeito de borda, incluindo os fluxos bióticos e abióticos. Por meio do índice de forma médio (MSI), pode-se determinar a tendência em relação à forma de um fragmento. Os fragmentos com formas mais circulares (MSI mais próximo a 1) estão menos sujeitos às intervenções da matriz, enquanto aqueles com formas 
mais alongadas (MSI maior que 1) são os mais sujeitos a essas intervenções e aos efeitos da borda interior (FORMAN; GODRON, 1986; SOUZA et al., 2014).

Para a paisagem do PEJ, o valor do MSI é de 2,48, demonstrando uma forma complexa e irregular (MCGARIGAL; MARKS, 1995). Quanto às Classes de uso e à cobertura do solo, as que apresentaram as formas menos irregulares foram Ag, com MSI de 1,53, e a Classe Ce, que obteve um MSI de 1,82. Inversamente, a Classe Cag, que corresponde aos corpos d'água, apresentou o maior MSI $(6,26)$, seguido das Classes Ve $(2,97)$ e Mg $(2,77)$, evidenciando formas bem irregulares e complexas. Na análise ponderada AWMSI, esse padrão se manteve, mesmo com a distribuição de pesos de acordo com o tamanho das áreas (Tabela 4).

Para Forman e Godron (1986), as paisagens naturais exibem formas complexas e irregulares, enquanto as paisagens antropogênicas tendem a possuir formas regulares e bordas retas, o que explica a Classe Ag ter apresentado o menor valor de MSI. Entretanto, as Classes Cag, Ve e Mg apresentaram formas mais irregulares justamente porque Cag representa os cursos d'água que, naturalmente, possui um formato mais alongado, ao passo que a irregularidade da $\mathrm{Ve}$ e da $\mathrm{Mg}$, localizados ao longo dos cursos d'água (Figura 1), está associada ao fato de que esses ecossistemas naturais formam corredores e apresentam alta relação perímetro/área. Porém, isso não significa que sua forma seja prejudicial às relações ecológicas (CABACINHA; CASTRO; GONÇALVES, 2010; SCHMIDT et al., 2011).

Apesar de o MSI demonstrar a circularidade ou a irregularidade do fragmento, podese inferir o quanto um fragmento ou classe está susceptível a manter a qualidade estrutural de um ecossistema (SOUZA et al., 2014). O isolamento do fragmento está condicionado a muitas especificidades, tais como a distância média entre os fragmentos, que influenciam os padrões de dispersão e a sobrevivência de espécies, bem como à capacidade de um organismo usar os recursos em fragmentos adjacentes (MCGARIGAL; MARKS, 1995). Portanto, a métrica MSI deve ser interpretada associada à métrica MNN, que apresenta o grau de proximidade dos fragmentos de mesma classe (CABACINHA; CASTRO; GONÇALVES, 2010).

A distância média do fragmento de mesma classe (MNN) mais próximo para todas as classes do PEJ foi de 457,8 m, o que, na concepção de Calegari et al. (2010) e Almeida (2008), demonstra um isolamento muito alto, sendo prejudicial aos fluxos gênicos entre populações de animais e vegetais. No entanto, como a métrica MNN está diretamente associada à área e à distância dos fragmentos (JUVANHOL et al., 2011), deve-se considerar que no PEJ a Classe Cag possui apenas três fragmentos e apresentou o maior MNN (13.143,2 m), o que alavancou o MNN para toda a paisagem.

Desconsiderando a Classe Cag por representar tão somente os corpos d'água que foram identificados na imagem Landsat-8 e com exceção das Classes Cam e Pr, com menores valores de MNN, as demais Classes apresentaram valores elevados de MNN, evidenciando um alto grau de isolamento, com destaque para a Classe Ag, cujo valor de MNN foi de 1.466,9 m (Tabela 4). Porém, para a Classe Ag, por serem áreas antrópicas dentro de uma unidade de conservação de proteção integral, o isolamento pode ser favorável à conservação das demais áreas.

Avaliando, ainda, os dados da Tabela 4, o valor do MNN e MSI da Classe Ve, de 258,9 m e 2,97 , respectivamente, ou seja, alto grau de isolamento e forma bastante irregular, denota certo grau de fragmentação dessa classe, bem como mostra que seus fragmentos estão mais susceptíveis aos efeitos de borda. Entretanto, os 175 fragmentos (NumP) dessa classe, encontrando-se isolados entre si, caracterizam-nos a servirem como trampolins ecológicos (stepping stones). Dos 57 fragmentos classificados como pequenos (5,01-10 ha), 44 pertencem à classe Ve, da mesma forma que dos 269 da classe fragmentos médios (10,01-100 ha), 101 também são de Ve (Tabela 3).

Apesar do alto grau de isolamento dos fragmentos do PEJ (a nível de classe), deve-se considerar que esses isolamentos podem ser caracterizados por fatores ambientais (por exemplo relevo, solo e água). É o caso das veredas, que são naturalmente isoladas pelas condições ambientais. Nas lições de Vieira et al. (2003), a existência de habitats de estrutura florestal no entorno dos fragmentos, que neste caso são os fragmentos de classes diferentes, mas vizinhos entre si (Figura 1), aumenta a conectividade entre os fragmentos, diminuindo o efeito prejudicial do isolamento. 


\section{Conclusão}

A vegetação existente no PEJ sofreu pouca alteração no período de 2000 a 2015 . A matriz da paisagem é composta pela fitofisionomia de Campo, com LPI de 35\%.

Apesar do aumento das áreas de Agropecuária, i.e., aumento de 0,20\% no período de 15 anos (2000-2015), esta classe continua representando menos de $1 \%$ de toda a área do PEJ. Ela, porém, exige atenção por parte dos gestores em razão do seu aumento. Quanto às demais classes, as poucas variações ocorreram na mudança dos tipos de classes entre si.

A paisagem do PEJ é fragmentada, mas seus fragmentos fazem parte do ecossistema local. Além disso, ela possui alta heterogeneidade espacial e uma forma complexa e irregular que favorece a biodiversidade e reafirma a condição natural das áreas do PEJ.

Devido à quantidade de pequenos fragmentos, o PEJ pode estar susceptível a alterações na dinâmica de sua paisagem, especialmente para as Classes $\mathrm{Mg}$ e Ve, demonstrando atenção por parte dos gestores da UC principalmente porque esses fragmentos pequenos possuem uma importante função, atuando como stepping stones.

Quanto aos fragmentos de mesma classe, eles se apresentaram com alto isolamento. Entretanto, deve-se considerar que os vizinhos de cada fragmento, independente da classe de uso e cobertura do solo, são áreas naturais e, portanto, fazem parte do ecossistema, o que proporciona maior conectividade, diminuindo os efeitos do isolamento.

\section{Agradecimentos}

O presente trabalho foi realizado com apoio da Coordenação de Aperfeiçoamento de Pessoal de Nível Superior - Brasil (CAPES) - Código de Financiamento 001. Agradecemos, ainda, ao Programa de Pós-Graduação em Engenharia Florestal da UFPR.

\section{Referências}

AGÊNCIA NACIONAL DE ÁGUAS (Brasil). Sistemas de informações hidrológicas-Hidroweb. Séries históricas. Brasília, [2017]. Disponível em: http://hidroweb.ana.gov.br/HidroWeb.asp?Toc Item=1080\&TipoReg=7\&=1\&SerieHist=true. Acesso em: 05 maio 2017.

ALMEIDA, C. G. Análise espacial dos fragmentos florestais na área do Parque Nacional dos Campos Gerais, Paraná. 2008. Dissertação (Mestrado em Gestão do Território) - Universidade Estadual de Ponta Grossa, Ponta Grossa, 2008.

ANDREAZZI, C. S.; PIRES, A.; FERNANDEZ, F. A. Mamíferos e palmeiras neotropicais: interações em paisagens fragmentadas. Oecologia Brasiliensis, [s. l.], v. 13, n. 4, p. 554-574, 2009.

CABACINHA, C. D.; CASTRO, S. S.; GONÇALVES, D. A. Análise da estrutura da paisagem da alta bacia do Rio Araguaia na savana brasileira. Floresta, Curitiba, v. 40, n. 4, dez. 2010.

CALEGARI, L. et al. Análise da dinâmica de fragmentos florestais no município de Carandaí, MG, para fins de restauração florestal. Revista Árvore, Viçosa, MG, v. 34, n. 5, p. 871-880, 2010.

ESTEVES PELUZIO, J.; FIEDLER, N. C.; ZANETTI, S. S. Análise da dinâmica espaço-temporal dos fragmentos florestais da sub-bacia hidrográfica do Rio Alegre, ES. Cerne, Lavras, v. 21, n. 2, p. 311-318, 2015.

FONSECA, L. M. G. Processamento digital de imagens. São José dos Campos: Instituto Nacional de Pesquisas Espaciais, 2000. 105 p.

FORMAN, R. T. T.; GODRON, M. Landscape ecology. New York: John Wiley \& Sons, 1986. 619 p. 
FRAGOSO, J. M. V. Home range and movement patterns of white-lipped peccary (Tayassu pecari) herds in the northern Brazilian Amazon. Biotropica, [s. l.], v. 30, n. 3, p. 458-469, 1998.

FROHN, R. C.; HAO, Y. Landscape metric performance in analyzing two decades of deforestation in the Amazon Basin of Rondonia, Brazil. Remote Sensing of Environment, [s. l.], v. 100, n. 2, p. 237-251, 2006.

JESUS, E. N. et al. Estrutura dos fragmentos florestais da bacia hidrográfica do rio Poxim-SE, como subsídio à restauração ecológica. Revista Árvore, Viçosa, MG, v. 39, n. 3, p. 467-474, 2015.

JUVANHOL, R. S. et al. Análise Espacial de Fragmentos Florestais: caso dos Parques Estaduais de Forno Grande e Pedra Azul, Estado do Espírito Santo. Floresta e Ambiente, Seropédica, v. 18, n. 4, p. 253-264, 2011.

MCGARIGAL, K.; MARKS, B. J. FRAGSTATS: spatial pattern analysis program for quantify Ingland scape structure: reference manual. Oregon: Oregon State University, 1995. 59 p.

MIRANDA, G. H. B.; FARIA, D. S. Ecological aspects of black-pincelled marmoset (Callithrix penicillata) in the Cerradão and dense Cerrado of the Brazilian Central plateau. Brazilian Journal of Biology, São Carlos, v. 61, n. 3, p. 397-404, 2001.

MUCHAILH, M. C. et al. Metodologia de planejamento de paisagens fragmentadas visando a formação de corredores ecológicos. Floresta, Curitiba, v. 40, n. 1, p. 147-162, 2010.

PEREIRA JÚNIOR, A. C. P. et al. Modelling fire frequency in a Cerrado savanna protected area. PloS one, [s.l.], v. 9, n. 7, p. e102380, 2014.

PIROVANI, D. B. et al. Análise espacial de fragmentos florestais na Bacia do Rio Itapemirim, ES. Revista Árvore, Viçosa, MG, v. 38, n. 2, p. 271-281, 2014.

REMPEL, R. S.; KAUKINEN, D.; CARR, A. P. Patch Analyst and Patch Grid. Ontario: Ontario Ministry of Natural Resources, Centre for Northern Forest Ecosystem Research, 2012. Disponível em: http://www.cnfer.on.ca/SEP/patchanalyst/Patch5_2_Install.htm. Acesso em: 10 jun. 2017.

RUDOLPHO, L. D. S. et al. Aplicação de técnicas de geoprocessamento e métricas da paisagem na análise temporal da cobertura florestal da Bacia do Ribeirão Fortaleza em Blumenau/SC. In: SIMPÓSIO BRASILEIRO DE SENSORIAMENTO REMOTO, 16., 2013, Foz do Iguaçu. Anais [...]. Florianópolis: INPE, 2013.

SANTOS, R. M. D. et al. Riqueza e similaridade florística de oito remanescentes florestais no norte de Minas Gerais, Brasil. Revista Árvore, Viçosa, MG, v. 31, n. 1, p. 135-144, 2007.

SCHMIDT, I. B. et al. Fogo e artesanato de capim-dourado no Jalapão - usos tradicionais e consequências ecológicas. Biodiversidade Brasileira, [s. l.], n. 2, p. 67-85, 2011.

SOUZA, C. G. et al. Análise da fragmentação florestal da área de proteção ambiental Coqueiral, Coqueiral-MG. Ciência Florestal, Santa Maria, v. 24, n. 3, p. 631-644, 2014.

TOCANTINS (Estado). Instituto Natureza do Tocantins. Plano de manejo do Parque Estadual do Jalapão: diagnóstico e planejamento. Palmas: Naturatins, 2003. 131 p.

TOCANTINS (Estado). Secretaria de Planejamento. Estudo da dinâmica da cobertura e uso da terra do Estado do Tocantins. Palmas: Seplan; DZE, 2012.

VIEIRA, M. V. et al. Mamíferos. In: RAMBALDI, D. M.; OLIVEIRA, D. A. S. (org.). Fragmentação de Ecossistemas: causas, efeitos sobre a biodiversidade e recomendações de políticas públicas. Brasília: MMA; SBF, 2003. p. 125-145.

XAUD, M. R.; EPIPHANIO, J. C. N. Dinâmica do uso e cobertura da terra no sudeste de Roraima utilizando técnicas de detecção de mudanças. Acta Amazonica, Manaus, v. 44, n. 1, p. 107-120, 2014. 